\title{
Die Melioration der aargauischen Reußebene in ganzheitlicher Sicht
}

Max H. Disteli

\begin{abstract}
"Wir versündigen uns deshalb, weil wir nur Teile des Lebens überdenken, das Ganze aber überdenkt niemand.»

Seneca
\end{abstract}

Was uns der römische Weise für das Leben zu bedenken gibt, das gilt wohl auch für die Landschaft, in die das Leben ja schicksalhaft eingebettet ist. Der nun folgende kleine Beitrag soll das bald zur Abstimmung kommende Reußtalgesetz unter einem gesamtlandschaftlichen Aspekt kritisch würdigen und werten, denn überall wo Ziele gesetzt werden, müssen wir eine Wertung vornehmen. Die Darlegungen mögen mit einer Schilderung des landschaftlichen Rahmens beginnen, wobei sich der Schreibende ganz an die Ausführung von Dr. H. U. Stauffer $\uparrow$, dem Erforscher der Flora der aargauischen Reußebene, hält. (Die aargauische Reußebene als Wohngebiet aussterbender Sumpf- und Wasserpflanzen, Sonderdruck aus: Bericht des Geobotanischen Instituts der ETH, Stiftung Rübel, 32, Heft 1960, Zürich 1961.) Hernach soll kurz und anhand von Beispielen die Pflanzen- und Tierwelt, die in erster Linie durch Kultivierungsmaßnahmen gefährdet ist, zur Sprache kommen, dann wird das Gesetz über den Hochwasserschutz, die Entwässerung und die Bodenverbesserung im Gebiet der Reußebene (das Reußtalgesetz) kurz dargelegt und anschließend kritisiert werden.

\section{Der landschaftliche Rahmen}

Südlich des aargauischen Städtchens Bremgarten dehnt sich auf einer Länge von mehr als 10 Kilometern und auf einer Breite von 2 bis 4 Kilometern auf beiden Reußufern von Mühlau im Süden bis an die Moränen von Hermetschwil und Zufikon im Norden die Reußebene. Die weite, fast flache Talsohle besteht im südlichen Teil aus Kiesablagerungen, denen teilweise Torf aufgelagert ist, im nördlichen Teil aus sehr feinem sandig-tonigem Gestein. Die Schotter der Talsohle sind strichweise grundwasserführend, jedoch längst nicht so reich wie z. B. im Wigger- oder Suhrental. Vom Fluß versickert Wasser in die Grundwasserströme des Tales. Die Siedlungen liegen meist am Talrand auf Terrassen oder Schwemmkegeln (Mühlau, Merenschwand, Aristau, Rottenschwil), einige Weiler und alte Ein- zelhöfe (Heftihof, Holzerhof) finden sich auf geringen Erhöhungen in der Ebene selbst. Die Gygersche Karte von 1667 zeigt die frei strömende Reuß mit vielen Armen, Inseln und Altläufen. Bei Hochwasser wechselte der Fluß dank seiner innern Dynamik oft stark seinen Lauf. So entstanden und schwanden im Wechsel Flußarme und Totläufe. Die Ebene war dem Menschen feindlich, war sie doch mit weiten Sumpf- und Riedflächen, mit Auen- und Bruchwäldern bedeckt, einem Dorado für zahlreiche Pflanzen- und Tierarten, besonders des amphibischen Biotops. Mit dem zunehmenden Bevölkerungsdruck, dem Landhunger, der Säkularisierung der menschlichen Ziele, der ständigen Vervollkommnung des Instrumentariums zur Beherrschung und Machbarkeit der Dinge setzten im letzten Jahrhundert die Anstrengungen zur Begradigung und Eindämmung der Reuß und zur Entwässerung des dahinter liegenden Landes ein. Es kam aber auch im 20. Jahrhundert noch zu Dammbrüchen und Überschwemmungen, so in den Jahren 1910, 1912, 1953. In regenreichen Jahren kann der Grundwasserspiegel so sehr steigen, daß auch Teile des Kulturlandes unter Wasser gesetzt werden. Heute hat das erfahrungsgemäß regelmäßig eine Flut von Anfragen und Interpellationen im Parlament zur Folge. Den "unhaltbaren» Zuständen soll dann jeweilen «endgültig» mit kostspieligen Maßnahmen abgeholfen werden.

Das bestehende Entwässerungsnetz setzt sich linksufrig aus zwei größeren Kanälen, dem Reußkanal und dem daraus abzweigenden Binnenkanal, zusammen. Zahllose kleinere und kleinste Stichkanäle fügen sich ergänzend der einfachen Entwässerung ein. Das Entwässerungsnetz entführt auch die Wasser der Bäche, die über den Osthang des Lindenbergs herabströmen. Die Gräben sind zum Teil mit prächtigen Bäumen, hauptsächlich Erlen, gesäumt. Die alten Reußläufe lassen sich vielfach noch nachweisen, teils besitzen sie noch offenes Wasser (Stille Rüß bei Rottenschwil, siehe Fig. 2, Ober- und Untersee, bei Althäusern und andere), teils zeigen sie Verlandungsstadien mit Schwingböden (Hellsee, Aristau, Schorenschachen), teils sind sie nur noch als Riedzonen im umgebenden Wies- oder Ackerland zu erkennen. Torfstiche bei Birri und Merenschwand haben zu weiteren offenen, stillen Wasserflächen geführt, die das parkartige Landschaftsbild verschönern und beleben. In Flußnähe 
werden kleinere Gebiete bis heute noch periodisch überschwemmt, und so finden sich dort noch Fragmente von Auenwald, so beim Geißhof, zwischen Rottenschwil und Werd und beim Heftihof. Die mannigfaltigen menschlichen, im Maße bleibenden Kultureinflüsse haben zusammen mit den naturgegebenen Voraussetzungen zu einer erstaunlichen Vielfalt an Lebensmöglichkeiten geführt, der ein entsprechender Reichtum an Pflanzen- und Tierarten gegenübersteht. Wir haben eine noch stark naturgeprägte, daher sehr schutzwürdige Kulturlandschaft mit allen Eigenschaften einer ausgesprochenen Erholungs- und Forschungslandschaft vor uns.

\section{Die Pflanzen- und Tierwelt der Reußebene}

(Nach Angaben und auf Grund einer Exkursion von und mit Herrn E. Kessler, Sekundarlehrer Oberrohrdorf.)

Die von H. U. Stauffer, dem allzufrüh verstorbenen und für den Aargau hochverdienten Botaniker, im Jahre 1958 begonnenen Untersuchungen haben ergeben, daß weit über 700 Arten von Gefäßpflanzen in der Talebene vorkommen, was gut der Hälfte der überhaupt im Kanton wachsenden Pflanzenarten entspricht. Dabei finden sich die bemerkenswertesten Funde unter den Wasser- und Sumpfpflanzen, jenen Arten also, die in ganz Europa wegen der Zerstörung ihrer Lebensstätten durch die alles erfassende technische Zivilisation im Aussterben begriffen sind und damit eine krankhafte Einförmigkeit der den Landschaftsraum füllenden Lebensformen bewirken. Den einzigartigen Pflanzengesellschaften entspricht eine mannigfaltige Tierwelt, vor allem Insekten, Spinnentiere, Lurche, Kriechtiere, Weichtiere und Vögel. Überdurchschnittlich gut ist in der deckungsreichen Landschaft auch der Jagdwildbestand. Dem flüchtigen Durchfahrer fallen Pflanzen und Tiere wohl weniger auf als die reich gegliederte Landschaft. Sie sind aber entscheidend wichtige Strukturelemente dieser noch relativ wasserreichen Landschaft. Wer den Naturelementen beobachtend und sinnend nachgeht, der erlebt Natur als Filigranarbeit und als wahres Kunstwerk.

Der Hellsee bei Aristau ist ein alter, vollständig verlandeter Reußlauf mit einer Entwicklung in Richtung Zwischenmoor. Einzelne Partien bilden Schwingrasen. Sphagnum ist in verschiedenen Arten anzutreffen, ferner Drosera rotundifolia, Polytrichum strictum, Menyanthes trifoliata, Carex inflata, Carex lasiocarpa, Carex Buxbaumii, Carex acutiformis nebst andern Scheingrasarten. Dryopteris Thelypteris (Moorschildfarn), Equisetum fluviatile, Luzula multiflora, Scirpus silvaticus, Alnus glutinosa gesellen sich den feuchtigkeitsliebenden Arten bei. Carex inflata oder rostrata bildet blaugrüne Rasen, während braune Flächen durch Vorherrschen von Juncus subnodulosus (Knotenbinse) geprägt werden. Später im Jahr übergießt das Pfeifengras (Molinia coerulea) die Rasen mit einem violetten Schimmer. An tiefen und feuchten Stellen, sehr abhängig von der Magerkeit des Bodens und der periodischen Überschwemmung, gedeiht in konzentrierten Beständen die herrliche Iris sibirica. Bei mehrmaligem Schnitt gehen die Bestände verloren. Die prächtige Pflanze reagiert sehr empfindlich auf Kunstdünger und Grundwasserabsenkung. Durch Kiesentnahme aus dem Reußbett ist der Wasserstand angrenzender Felder so gesunken, daß die Bauern sie unter den Pflug genommen und auf diese Weise große Bestände der blauen Schwertlilie zugrunde gerichtet haben. Da die Lilie während der Blütezeit recht auffällig ist, wird sie auch durch massenhaftes Pflücken sehr geschädigt, da zu wenig Fruchtstände ausreifen können. Die Motorisierung der Besucher und eine Wegerschließung für die hintersten Flächen wirken sich verheerend aus, da der Mensch nach wie vor oft nicht als stiller Bewunderer und als Ehrfürchtiger auftritt, sondern als gieriger und gedankenloser Plünderer. Wenn gar zu Geschäftszwecken hier Schnittblumen geholt werden, kann man sich den Grad der Bedrohung dieser Charakterpflanze des Reußtales ausmalen. Ohne Aufsicht sind die besten Verordnungen wertlos, wirken sich doch Bevölkerungsdruck und oberflächlicher Erholungsrummel gerade in diesen nichtwirtschaftlichen Bereichen höchst nachteilig aus.

Der Birriweiher ist aus einer alten Torfgrube entstanden. Er gilt wegen der zahlreich hier brütenden oder durchziehenden Vogelarten als Naturschutzobjekt. Unter den etwa 36 festgestellten Arten sind Rohrammer, Rohrdommel, grünfüßiges Teichhuhn, Teichrohrsänger, Heuschreckenrohrsänger, großer Raubwürger und Graureiher zu nennen. Der mächtige, unterseits hellfarbene Fischadler macht hier als Durchzüger oft längere Zeit Aufenthalt. DerWeiher bildet zusammen mit seiner Umgebung, die leider immer intensiver gedüngt und genutzt wird, auch ein Landschaftsschutzobjekt.

Der Schorenschachen nordnordöstlich von Mühlau etwa 28 ha groß, ist naturkundlich ein ganz hervorragendes Reservat, das Kleinseggenrieder mit einer reichen Orchideenflora (Parvo-Caricetum) sowie Filipenduleten (Rüsterstaudengesellschaften) aufweist. Durch Grundwasserinfiltration von der Reuß her erkennen wir hier eine besondere Begünstigung der feuchtigkeitsliebenden Flora. Hier wohnen denn auch zwei Charaktervögel der Reußebene: einmal der große Brachvogel, der mit seinem weithinhallenden Flötenpfiff und seinem Triller einen besonderen Ton in die sommerliche Landschaft fügt. Dieser schöne Vogel - ein wahres Naturdenkmal - hat 


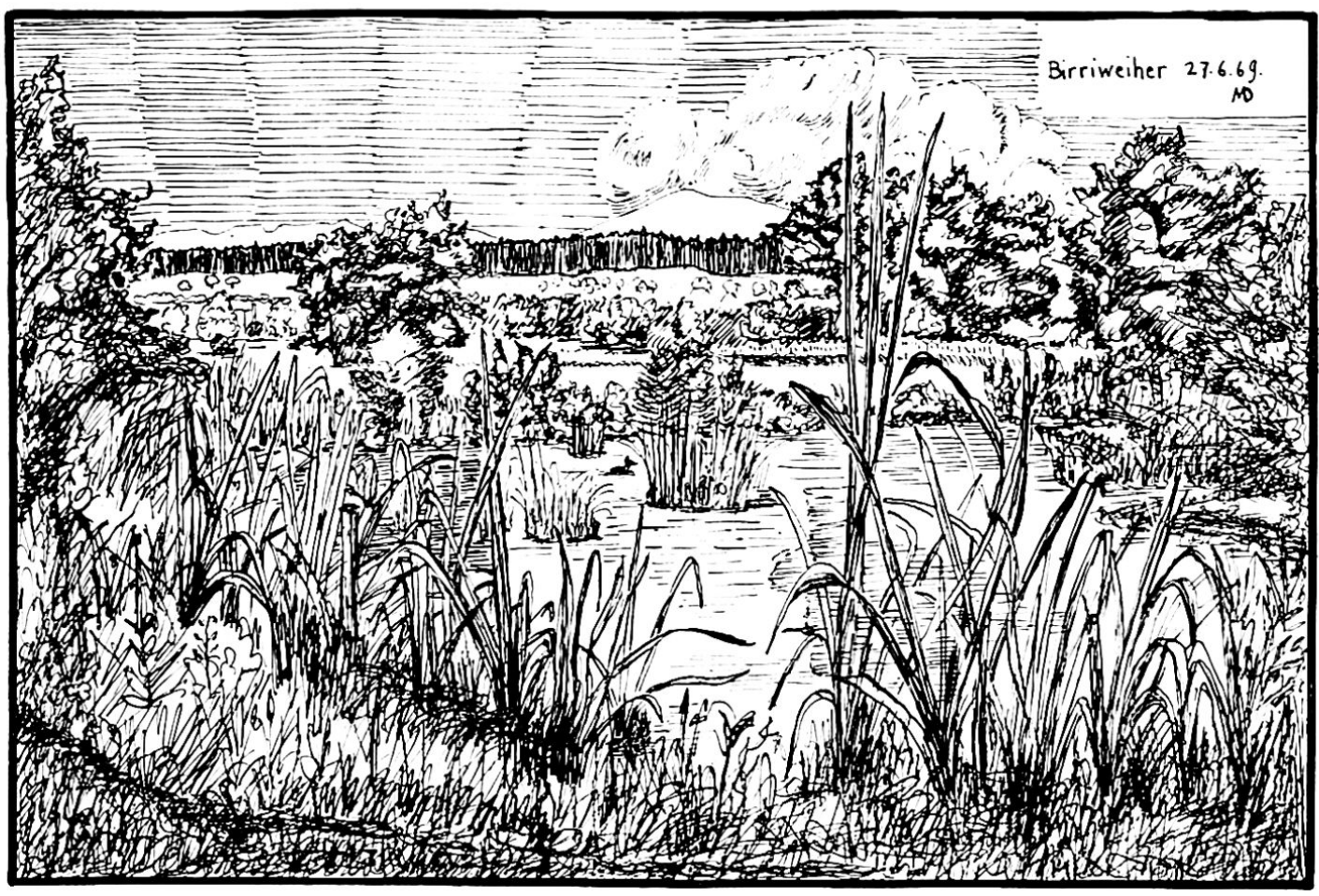

Figur 1. Der aus einer alten Torfgrube entstandene Birriweiher gilt wegen der zahlreich hier brütenden oder durchziehenden Vogelarten als Naturschutzobjekt

einen langen, leicht gebogenen Schnabel und kann seine Nahrung nur in Feuchtriedwiesen mit hohem Grundwasserstand finden und aufnehmen. Als weiterer Bodenbrüter haust der etwa krähengroße Kiebitz in einem unkultivierten Gebiet. Bei meinem Kontrollgang am 23.5.69 kreisten drei dieser Vögel über mir und stießen so lange ängstliche Warnrufe aus, bis ich das Brutrevier wieder verlassen hatte. $\mathrm{Da}$ die Reußebene botanisch, zoologisch, landschaftlich über die angrenzenden Kantone, den Kanton Aargau, über die ganze Schweiz hinaus, europäische Geltung beanspruchen darf, geht daraus hervor, daß sich der World Wildlife Fund auf internationaler Basis aktiv zum Schutze dieser Werte eingeschaltet hat, damit durch möglichst große Landkäufe naturnahe Landschaftsteile bewahrt werden können.

\section{Das Reußtalgesetz}

wurde im Entwurf am 23.11.67 vom Regierungsrat des Kantons Aargau dem Großen Rat zur Lesung vorgelegt. In äußerster Kürze zusammengefaßt, sieht es vor, im Interesse der Landwirtschaft und zwecks Gewinn von Kulturland (Teilweise Ersatz von durch Überbauungen aller Art verlorengegangenem Kulturland) die aargauische Reußebene im Halt von 4641 ha großteils zu entwässern, durch Dammbauten vor zukünftigen Hochwassern des Flusses abzusichern, die zahlreichen zerstreuten Landparzellen zusammenzulegen, ein neues, etwa $190 \mathrm{~km}$ langes Wegnetz anzulegen sowie die Gründung von etwa 60 neuen Hofsiedlungen zu ermöglichen. Auf die Belange des Natur- und Landschaftsschutzes soll nach Möglichkeit Rücksicht genommen werden. Insbesondere soll die Schaffung von Reservaten vorgesehen sein. Bestandteil der Melioration soll auch der Ausbau des Kraftwerkes Zufikon werden, obwohl Flußkraftwerke heute nur noch unter besonderen Umständen gebaut werden. Der Aufstau der Reuß soll eine starke Absenkung des Grundwassers und eine Schmälerung des lebenswichtigen Wasserkapitals verhindern. Die schlimmen Auswirkungen der Gradlegung des Oberrheins und des Rheinseitenkanals in der oberrheinischen Tiefebene sprechen eine deutliche Sprache. Mit dem Höherstau darf aber erst begonnen werden, nachdem die Oberlieger ihre Abwasser durch Kläranlagen gereinigt in den Fluß abgeben. Das betrifft vor allem einige Betriebe in den Kantonen Luzern und Zug. Ein Zweckentfremdungsverbot soll schließlich verhindern, daß das Land, das mit erheblichen Mitteln der Öffentlichkeit vor Überschwemmungen geschützt und einer intensiveren landwirtschaftlichen Nutzung zugeführt worden ist, der Landwirtschaft wieder entzogen werden kann.

\section{Würdigung und Kritik des Reußtalgesetzes}

Das voranschlagmäßig etwa 60 Millionen Franken kostende und etwa 20 Jahre Bauzeit in Anspruch 
nehmende Werk (Übersicht Fig. 3) ist ein Ergebnis des allgemein herrschenden Trends nach Perfektionierung, Mehrertrag, bequemerer Arbeitsweise, gröBerer Arbeitsproduktivität starrer Begrenzungen, Zeugnis des Zuges nach möglichst gleichen Wettbewerbsbedingungen in der Landwirtschaft, dann ein Resultat des immer stärker werdenden Bevölkerungsdrucks, der anhaltenden Hoch- und Überschätzung und der Priorität des Ökonomischen und Machbaren in der Werteordnung, nicht zuletzt auch die Folge ehrgeiziger, vom Fortschrittsgeist beseelter Politiker und Wirtschaftsführer der Region, Männer, die nicht müde werden, das "Beste» für ihre Talschaft zu fordern, um sie voll an den Segnungen des Wohlstandes teilhaben zu lassen. Dem einen oder andern wird gewiß ein Denkmal gesetzt werden.

In einer Gesamtsicht müssen wir den landschaftlichen Fragen im Gesetz besonders gründlich nachspüren. Der Gesetzestext hält fest, daß der Hochwasserschutz und die Melioration zweifellos eine gewisse Veränderung des Landschaftsbildes zur Folge haben und auf Flora und Fauna von Einfluß sein werden. So können sich die Maßnahmen wie folgt auswirken:

Jetzt bestehende allmähliche und unscharfe Grenzen von Räumen und Biobeständen werden nicht mehr geduldet und beseitigt. Scharfe und gerade Grenzen, die durch ein neues Wegnetz und durch rechteckige Parzellen geschaffen werden müssen, beeinträchtigen und banalisieren die im Gesetzesentwurf ausdrücklich festgestellte selten schöne Parklandschaft. Der Vorgang, der bei allen Regulierungen unfehlbar statthat, führt zur Monotonie und hebt jede Isolation, auch die der zu schaffenden Reservate, auf. Die auch vom Eidgenössischen Departement des Innern in der Botschaft an den Bundesrat vertretene Fläche von 250 ha Naturschutzgebieten, die mit Bundesmitteln zu 30\% subventioniert werden soll, nimmt sich sehr bescheiden aus, wenn man noch bedenkt, daß es sich um ein Objekt von nationaler Bedeutung handelt, wenn man weiter bedenkt, daß ein höchst wichtiger, im Gebiet von Ballungszentren liegender, nicht mehr herzustellender Erholungsraum zur Debatte steht. Verfolgt man die überaus großzügige internationale Entwicklungspolitik des Bundes, so muß man nur staunen, wie wenig die Zukunft für uns in der Schweiz kosten darf. Der Bundesbeitrag wird zudem nur dann gewährt, wenn der Kanton 50\% gibt. Den Rest sollen Privatorganisationen aufbringen. Die vorgemerkten Reservate liegen fast ausnahmslos flußnah zu beiden Seiten des Hochwasserdammes und am Süd- und Nordrand der Ebene, dort wo am ehesten ein genügend hoher Wasserstand gehalten werden kann, aber jedenfalls nur bei einer Verwirklichung des Kraftwerkes Zufikon. Im Weichbild der Talau sind die Reservate umstritten und zu klein, als daß sie das Landschaftsbild künftig noch wirklich mitzuprägen vermöchten. Sie sind als Feuchtgebiete durch die vorzunehmende Grundwasserabsenkung verloren und einer starken Versteppung durch die Strukturänderungen der Umgebung ausgesetzt (Hellsee, Obersee, Untersee, Stille Reuß usw.). Bereits ausgearbeitete Güterregulierungsprojekte haben unmißverständlich gezeigt, daß das Schachbrettmuster in einer Ebene dem Ingenieur unumstößlich erscheint, um die geforderte Rationalisierung in der Bewirtschaftung überhaupt verwirklichen zu können. So ist anzunehmen, daß die markanten Baumreihen, die sich ungeometrisch bestehenden gewundenen Entwässerungsgräben und kleinen Hangversteilungen entlang ziehen, fallen werden. Eine gut gemeinte Neubepflanzung der zukünftigen technischen Elemente in Rechteckformen kann dem Land keine Seele geben, keine Eigenart prägen und kann es mit den Einheitssiedlungshöfen zusammen unter Umständen zur Dutzendware stempeln.

Ferner ist festzuhalten, daß auch das Zweckentfremdungsverbot im Wortlaut so stark verwässert ist, daß einer späteren Industrialisierung des Gebietes prinzipiell nichts im Wege steht, daß im Gegenteil die wichtigsten Vorarbeiten für sie dann gemacht sind (Festigung des Bodens, Wegnetz). An diesem Tatbestand ändert auch die Rückerstattungspflicht der aufgewendeten Bodenverbesserungsbeiträge nichts.

Der Aufstau der Reuß, der ja schon jetzt besteht, aber verstärkt werden soll, hat Vor- und Nachteile. Der Hauptvorteil liegt wohl in der Wasserrückhaltung und Verhinderung der Tiefenerosion mit verheerend wirkender Dränage. Die Energieerzeugung kann von 12 auf etwa 100 Millionen Kilowattstunden im Jahr erhöht werden. Der entstehende Stausee wird für Pflanzen und Tiere neue Lebensmöglichkeiten bieten und besonders die unten gelegenen Reservate begünstigen. Andrerseits ist ein Aufstau auch ein schwerwiegender Eingriff in die natürlichen Bedingungen eines Flusses. Die Lebewelt des Wassers wird artenärmer, die schleichende Fließbewegung dichtet das Flußbett ab und beinträchtigt mit der Zeit die Infiltration zum Grundwasser hin. Ein aufgestauter Fluß verliert nicht nur an Selbstreinigungskraft, sondern spricht auch das Gemüt des Erholung suchenden weniger an, als ein kraftvoll dahinströmender. Im Ganzen gesehen und beim Abwägen müßten aber doch die Vorteile des Aufstaus die Nachteile weit überwiegen. Wir müssen uns darüber klar sein, daß wir nur mehr oder weniger große Übel wählen können, wenn wir so tief in den Landschaftshaushalt eingreifen und konstruktive Ordnung an Stelle von organischer setzen.

Wir müssen uns abschließend noch einmal ganz grundsätzlich mit dem geplanten Meliorationswerk auseinandersetzen: 


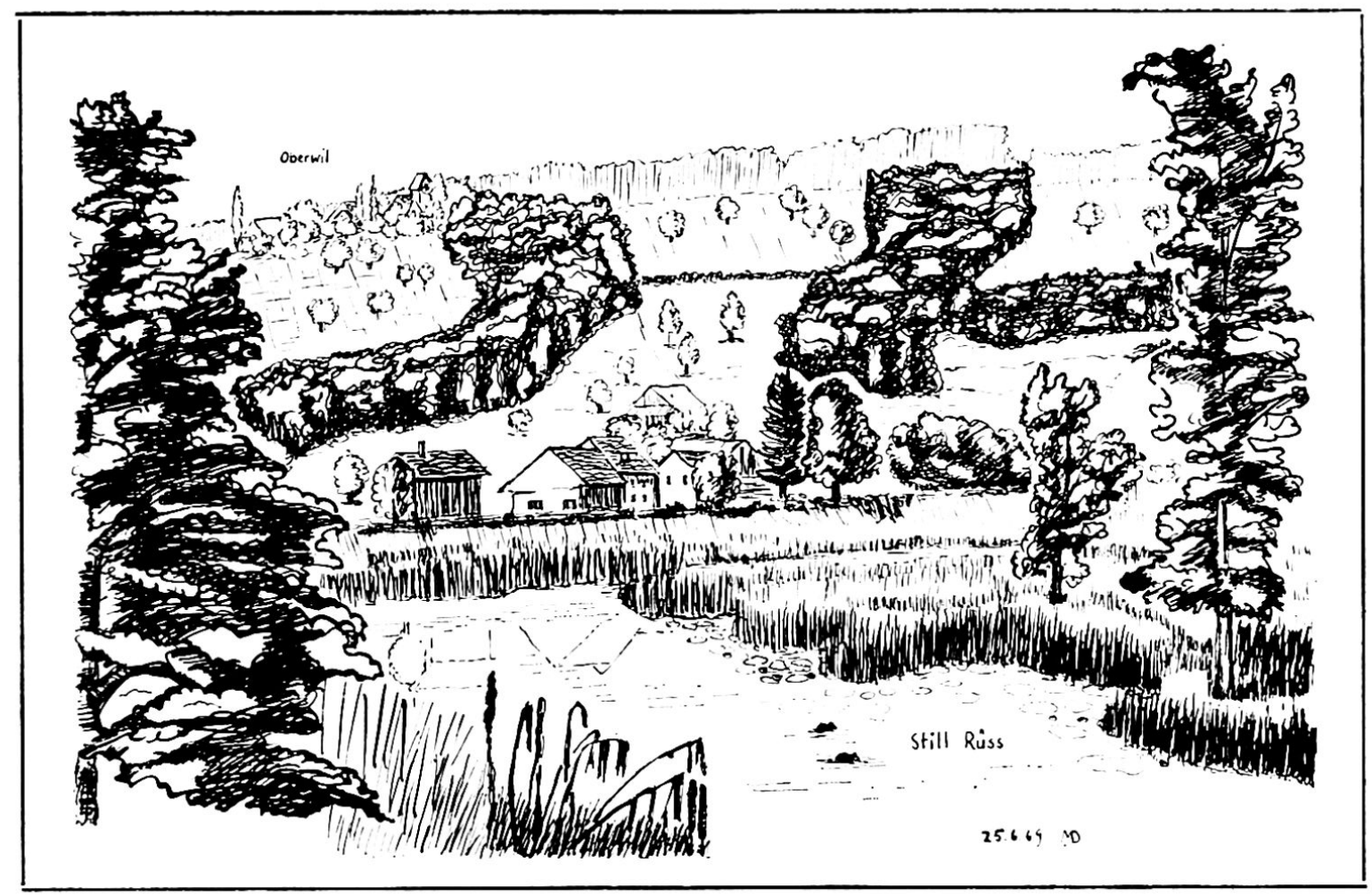

Figur 2.

Die «Still

Rüß» (Stille

Reuß), ein

noch offen

als Weiher

daliegendes

Altwasser

des Flusses

bei Rot-

tenschwil

Sicher ist, daß eine Kulturlandschaft sich nur dann pfleglich erhalten läßt, wenn bäuerliche Menschen sie bebauen und maßvoll nutzen. Damit der Bauer seinem Stande treu bleibt, wird versucht, durch massive Strukturverbesserungen ihm ein den übrigen Bevölkerungsschichten der Industriegesellschaft adäquates Geldeinkommen zu verschaffen. Das ist bis jetzt trotz Aufstockung und "Gesundschrumpfung» nicht gelungen und wird auch nicht gelingen, weil die Industriegesellschaft durch Tiefhaltung der Lebensmittelpreise, vor allem der Erzeugerpreise, einseitig Nutzen aus den Urproduzenten zieht. Die sogenannte Agrarfrage, d. h. die völlige Einschmelzung des Bauernstandes in die Industriegesellschaft, ist mit betrieblichen Rationalisierungen und Rationalisierung der Landschaft zumindest allein nicht zu lösen (Symptome sind Úberschüsse, Preiszusammenbrüche, Stützungsaktionen, Aufgabe von immer mehr Betrieben, Schwächung dadurch des politischen Einflusses der Bauernsame usw.). Durch einseitige ökonomistische Überlegungen und Maßnahmen in der Landwirtschaft werden andrerseits nichtwirtschaftliche Faktoren allzu stark vernachlässigt. Es wird auch übersehen, daß der Bauernhof kein Industriebetrieb ist, da er organisches Leben pflegt und mit den Zufälligkeiten des Wetters zu rechnen hat, während ein Industriebetrieb tote Güter im Haus herstellt.

Wir dürfen illusionslos sagen, daß heute eine Melioration, werde sie durchgeführt wo sie wolle, eine unerhört nivellierende Wirkung auf die Landschaft hat, sie ausräumt und zur Kultursteppe umprägt. Damit wird auf kurze Zeit zwar der Landwirtschaft geholfen, was zweifelsfrei sehr wichtig ist, gleichzeitig aber die gegenwärtig mindestens so wichtige Erholungsfunktion eines Gebietes abgewertet, was bei früheren landwirtschaftlichen Betriebsformen nicht der Fall war. Es frägt sich doch, ob nicht zu Gunsten der Erhaltung, ja landschaftlichen Aufwertung andere Wege und bessere Kompromisse gefunden werden könnten, als solche mit einigen noch nicht einmal gesicherten Naturschutzflecken in einer sonst zukünftig banalen und lebensschwachen Landschaft. Das Wirtschaftliche wird einfach zurzeit zu hoch bewertet, das Nichtwirtschaftliche zu niedrig. Wie wäre es, wenn die Hälfte Landes mit öffentlichen Mitteln aufgekauft würde, Halbkulturland (eminent wichtig z. B. für Wildhege!) und Naturschutzfläche mit besonderen Nutzungsservituten bliebe, während die übrige Hälfte, ohne Schematismus und nur nach Notwendigkeit zusammengelegt würde, ohne daß Großentwässerungen vorgenommen würden. Die bestehenden Entwässerungsanlagen könnten erneuert, etwas erweitert und leistungsfähiger gebaut werden, ohne daß ein Perfektionismus riesige Geldmittel verschlänge. Die ab und zu auftretenden Hochwasser, die für den Wasser-, Lebens- und Bodenhaushalt auch günstige Auswirkungen haben, könnten in ihren schädlichen Folgen bei den Bauern mittels öffentlicher Mittel und unter Einsatz einer neutralen Schätzungskommis- 
sion abgegolten werden. Das sind allerdings Vorschläge gegen die sogenannten Realitäten, aber sie sollen doch hier einmal genannt werden, da die verschiedenen Kommissionen, die sich mit landschaftlichen Fragen befassen, bis hinauf zur eidgenössischen Natur- und Heimatschutzkommission zum vornherein zur Totalmelioration "ja» sagten und wohl wirklich freie Vorschläge oder gar eine Ablehnung einer Totalmelioration weder wagen wollten noch konnten. Jede Art von Macht kann nämlich dazu eingesetzt werden, um das Verhalten der Schwächeren im Interesse der Stärkeren zu verändern. Rationale Begründungen für das Verhalten folgen erst, nachdem die herrschende Macht die
Verteilung der Rangnummern unausgesprochen vorgenommen hat. Die große Problematik wirtschaftlicher Macht wird selten richtig erkannt und dieser Macht auch zu Gunsten der Menschenwürde entschlossen entgegengetreten. Zum Abschluß möge versöhnend gesagt werden, daß die grundsätzlich bei solchen großen Werken auftretenden Meinungsverschiedenheiten im Grunde immer ethischer und religiöser Art, niemals aber sachlicher und wissenschaftlicher Art sind. Wo Ziele gesetzt werden müssen, wo Unwiederbringliches erhalten werden soll, da hört die Wissenschaft auf, und da fängt das Metaphysische an, die Frage nach dem Sinn des Lebens und allen Tuns. 